\title{
Erratum to: Decision-Making in Anterior Shoulder Instability
}

\author{
Michel P. J. van den Bekerom, Derek F. P. van \\ Deurzen, Karin M. C. Hekman, Olivier Verborgt, \\ Klaus Bak, Marco Brioschi, Chiara Fossati, \\ Riccardo Compagnoni, Alessandra Menon, \\ Hassanin Alkaduhimi, and Pietro Randelli
}

\section{Erratum to: \\ Chapter 16 in: G.M.M.J. Kerkhoffs et al. (eds.), ESSKA Instructional Course Lecture Book, https://doi.org/10.1007/978-3-662-56127-0_16}

The spelling of the author's name was wrong in chapter 16. The correct name is: Marco Brioschi

The updated original online version for this chapter can be

found at https://doi.org/10.1007/978-3-662-56127-0_16

M. P. J. van den Bekerom $(\bowtie) \cdot$ D. F. P. van Deurzen Orthopaedic Department, Onze Lieve Vrouwe

Gasthuis, Amsterdam, The Netherlands

Orthopaedic Department, Jan van Goyen Medical

Centre, Amsterdam, The Netherlands

e-mail: bekerom@gmail.com

K. M. C. Hekman

Interdisciplinary Center For Shoulder Rehabilitation,

IBC Amstelland, Amstelveen, The Netherlands

Orthopaedic Department, Jan van Goyen Medical

Centre, Amsterdam, The Netherlands

O. Verborgt

Orthopedic Department, AZ Monica,

Deurne, Belgium
K. Bak

Aleris Hamlet Parken, Copenhagen, Denmark

M. Brioschi · C. Fossati · R. Compagnoni

A. Menon $\cdot$ P. Randelli

1st Department, Azienda Socio Sanitaria Territoriale

Centro Specialistico Ortopedico Traumatologico

Gaetano Pini-CTO, Milan, Italy

Department of Biomedical Sciences for Health, University of Milan, Milan, Italy

H. Alkaduhimi

Orthopaedic Department, Onze Lieve Vrouwe

Gasthuis, Amsterdam, The Netherlands 